\title{
Bed nets used to protect against malaria do not last long in a semi-arid area of Ethiopia: a cohort study
}

Tarekegn Solomon ${ }^{1,5^{*}} \mathbb{0}$, Eskindir Loha ${ }^{1}$, Wakgari Deressa², Meshesha Balkew ${ }^{3}$, Taye Gari ${ }^{1,5}$, Hans J. Overgaard ${ }^{4}$ and Bernt Lindtjørn ${ }^{5}$

\begin{abstract}
Background: Long-lasting insecticidal nets (LLINs) are a key tool for malaria prevention and control. Currently, the recommended serviceable life of an LLIN is 3 years under field conditions. However, field studies show considerable variation in LLIN lifespan, from less than 2 years to more than 4 years. This study aimed to determine the attrition, physical integrity, functional survival, and bio-efficacy of LLINs under field conditions in south-central Ethiopia.

Methods: In October 2014, 7740 LLINs (PermaNet ${ }^{\circledR}$ 2.0) were distributed to 3006 households. Among the distributed LLINs, a cohort study involving 1532 LLINs in 659 households was carried out from October 2014 to November 2016. Data were collected every 6 months by observation, and through interviews with the heads of households. The proportional hole index was used to categorize LLINs as either serviceable or torn. In addition, 120 randomly selected LLINs were tested for bio-efficacy.

Results: The overall attrition of LLINs was $96 \%(n=993)$ during the study period. The nets' attrition was mainly due to disposal $(64.2 \% ; n=638)$. The proportion of LLINs with a hole size $0.5 \mathrm{~cm}$ or larger was $79.5 \%$ after 24 months. The use of the net on the previous night and having a clean net were associated with a good physical integrity. However, living in a household more than $1 \mathrm{~km}$ away from the mosquitoes' breeding site was associated with poor physical integrity. By the 24th month, only $4 \%$ of the nets met the criteria for functional survival. The median functional survival time of the nets was 12 months. A longer functional survival was associated with having a clean net, and shorter survival was associated with living in a household more than $1 \mathrm{~km}$ away from the mosquitoes' breeding site. The PermaNet ${ }^{\circledR}$ 2.0 met the criteria of effective bio-efficacy up to month 24 after distribution.
\end{abstract}

Conclusions: The study showed that the median serviceable life of LLINs is only 12 months. However, the bioefficacy of the LLINs is acceptable for at least 24 months. Therefore, stronger and more efficient LLINs need to be developed for conditions similar to those studied here.

Keywords: Durability, Long-lasting insecticide nets, Attrition, Physical integrity, Functional survivorship, Ethiopia

\footnotetext{
*Correspondence: tarekegn2solomon@yahoo.com

${ }^{1}$ School of Public and Environmental Health, Hawassa University,

Hawassa, Ethiopia

Full list of author information is available at the end of the article
} 


\section{Background}

Globally, the burden of malaria has declined in the past 15 years with the scaling-up of cost-effective vector control interventions, diagnosis, and treatment [1]. The reduction in the global incidence of malaria is estimated to be $37 \%$, and the decline in malaria-specific mortality is estimated to be $60 \%$ [1]. Similar reductions have also been observed in Ethiopia [2], but the incidence of malaria is still high; it is estimated that 2,588,000 cases of malaria and 5000 malaria-specific deaths occurred in 2016 [3].

Vector control through the use of long-lasting insecticidal nets (LLINs) is a widely implemented tool for the prevention of malaria $[4,5]$. To maximize the impact of the intervention, universal access to and use of LLINs by people at risk for malaria must be maintained [6]. However, access to LLINs remains lower than expected [3]. For example, in sub-Saharan Africa, only $43 \%$ of people had access to sufficient LLINs (a net for two people), and only $54 \%$ people slept under LLINs in 2016 [3]. According to a 2015 national malaria indicator survey, $64 \%$ of Ethiopian households own at least one LLIN, and 32\% have one LLIN for every two persons. The same survey reported that only $40 \%$ of the population at risk slept under a LLIN the night before the survey [7].

The LLIN interventions have a limited service life because they become worn out or are lost. The most common causes for the short service life of LLINs are a high attrition rate and physical damage [8-12]. Moreover, care and repair of bed nets, usage pattern, washing frequency, and type of LLIN all have potential impacts on the length of the service life of an LLIN [8, 13-15]. The World Health Organization (WHO) recommends that LLINs should be serviceable for at least 3 years under field conditions, with adequate insecticidal activity [16]. However, studies show considerable variation in the length of an LLIN' serviceable life, ranging from less than 2 years to more than 4 years $[8,13,17-19]$. Furthermore, it cannot be assumed that an LLIN product that is durable in one setting will last in other settings. Thus, there is substantial need for regional data to assess the durability of LLINs [20, 21]. Such data could inform decision-makers regarding how often bed nets should be distributed. Furthermore, understanding the factors that lead to a shortened LLIN service life could help guide communication interventions for behavioural change [20].

Previous studies from Ethiopia have investigated physical integrity and bio-efficacy of LLINs using cross-sectional study design $[17,21,22]$. However, these studies did not consider the attrition rate, functional survival, or potential causes of poor physical integrity of LLINs. To fill this knowledge gap, this study used a cohort design to determine the durability of LLINs under user conditions in the Adami Tullu district, south-central Ethiopia, in terms of attrition, physical integrity, functional survival, and bio-efficacy.

\section{Methods \\ Study setting}

This study was carried out in the Adami Tullu district in south-central Ethiopia (Fig. 1) from October 2014 to November 2016. The district is located approximately $160 \mathrm{~km}$ south of Addis Ababa. The study population was primarily composed of the Oromo ethnic group, who follow the religion Islam. This rural population primarily engages in farming, livestock, and fishing. Based on the 2007 national census, approximately 190,000 people lived in the district in 2017 [23]. The district has 48 kebeles (the lowest government administrative unit), each with an average population ranging from 1000 to 5000 people [23].

Malaria is among the leading causes of illness in the Adami Tullu district, sometimes occurring as an epidemic [24]. The shores of Lake Zeway and irrigated areas serve as mosquito breeding sites in the district $[25,26]$. Anopheles arabiensis is the main malaria vector, whereas Plasmodium falciparum and Plasmodium vivax are the main parasites of malaria in the district [27, 28]. The district is a drought-prone area, and is characterized by a semi-arid climatic condition [29]. The district was affected by a severe drought that occurred in 2015 following the El Nino [30]. Effects of the drought included food shortages, a decline in annual rainfall (by 60\% in 2015) and an increase in the average maximum temperature $\left(2^{\circ} \mathrm{C}\right.$ above normal $)$ [31].

\section{Study design and participants}

This study was part of a cluster-randomized controlled trial that aimed at quantifying the combined effect of indoor residual spray (IRS) and LLINs against clinical malaria, compared with LLINs or IRS alone or routine intervention (the MalTrials project) [32]. The trial had 176 study clusters (44 clusters per arm). The LLIN+IRS arm contained 1619 households and 8216 people; the LLIN-alone arm contained 1387 households and 7288 people; the IRS-alone arm contained 1530 households and 7753 people; and the routine arm contained 1544 households and 8038 people. In early October 2014, 7740 LLINs (purchased in June 2014 from the Vestergaard Frandsen Group) were distributed to 3006 households, both in combination and in the LLIN-alone arm. They had a light blue colour and rectangular shape, with a size of $160 \mathrm{~cm}$ width, $180 \mathrm{~cm}$ length, and $150 \mathrm{~cm}$ height [32]. The distribution of LLINs was conducted based on the National Malaria Guidelines: one net for a family with 1-2 persons; two nets for a family with $3-5$ persons; 


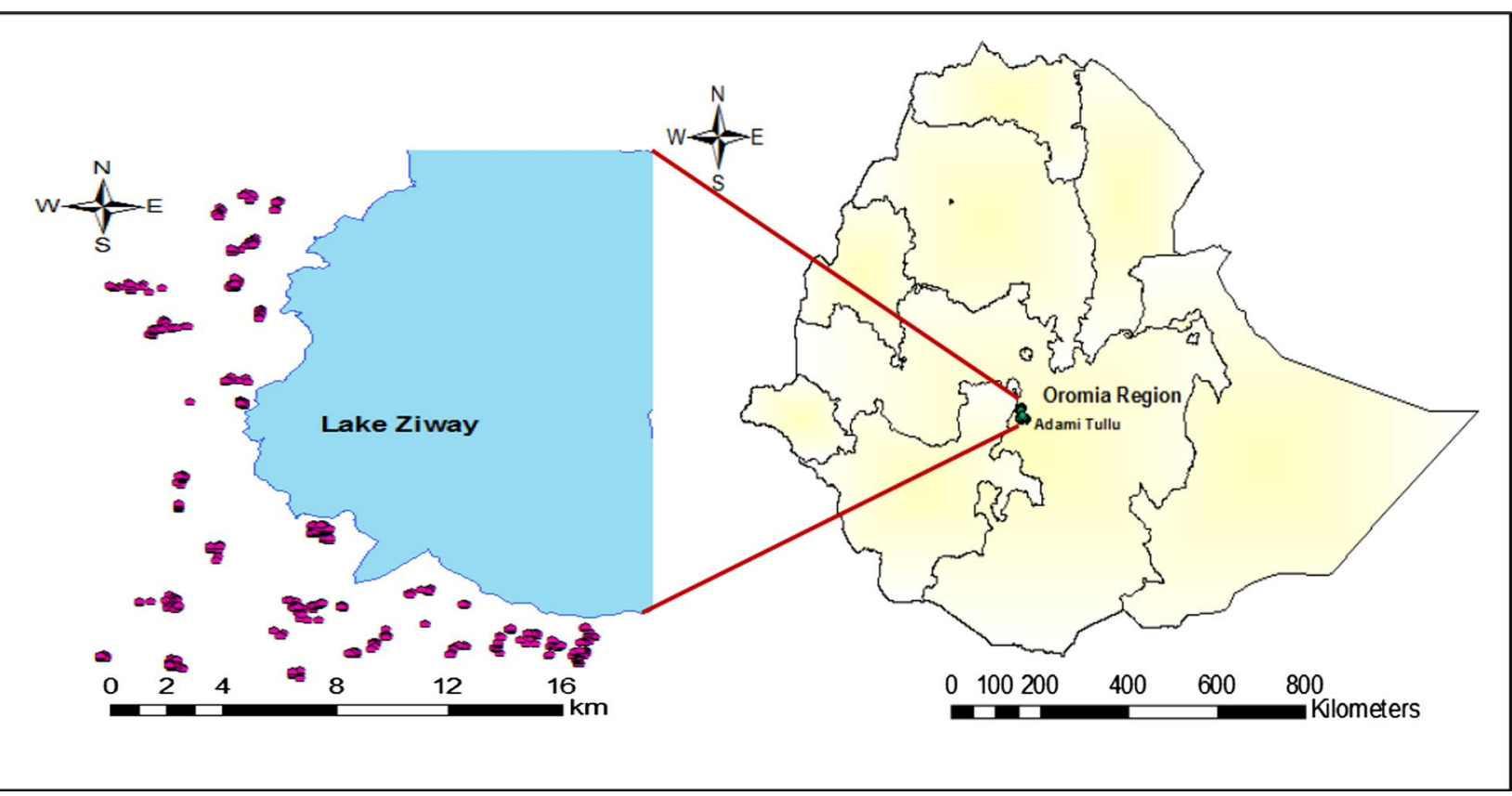

Fig. 1 Map of the study area showing the location of selected households in south-central Ethiopia

three nets for a family with 6-7 persons; and four nets for a family with $\geq 8$ persons [33]. A "hang-up" campaign and net tracking activities were carried out after distribution by putting a unique identification number on each LLIN with indelible ink.

Using the malaria trial framework [32], a cohort study was conducted among households with newly distributed LLINs to assess attrition, physical integrity, and functional survivorship. Four follow-up surveys were conducted every 6 months. The first survey was conducted in April 2015, the second in October 2015, the third in April 2016, and the fourth in early November 2016. The LLINs were followed until one of the following outcomes: LLIN loss due to discarding, distraction, used for other purposes, given away to other users, sold, stolen, lost to follow up, torn, or to the end of the study. Moreover, every 6 months cross-sectional surveys were carried out to evaluate the ability of LLINs to knockdown (KD) or kill susceptible Anopheles mosquitoes (bio-efficacy).

\section{Sample size estimation}

The sample size was calculated based on the findings from a study in Benin, in which $48 \%$ of the LLINs were in poor condition (torn) after 1.5 years of use [13]. Using a single-population proportion formula (with OpenEpi software), and assuming a $4 \%$ margin of error, a $95 \%$ confidence level at $\alpha=5 \%$, and a $10 \%$ non-response rate, a total sample size of 659 LLINs was calculated. The households were randomly selected from a sampling frame of the LLIN-alone arm of the trial. Computer-generated random numbers were used to select random samples of LLINs using IBM SPSS version 20.0 (Armonk, NY: IBM Corp. USA). To avoid selection bias, all LLINs in the selected households were included in the study. A total of 1532 LLINs in 659 households were enrolled at baseline for evaluation of attrition and functional survivorship. A sub-sample of 833 LLINs were enrolled at the sixth month and followed for assessment of physical integrity. According to WHO recommendations [34], a total of 120 LLINs (30 LLINs per survey) were evaluated for bio-efficacy over a 2-year period. The LLINs were collected for the test based on the eligibility criteria of being used for sleeping during data collection. One LLIN per household was considered for the test. The LLINs that were taken for analysis were immediately replaced with new LLINs.

\section{Data collection}

Baseline and follow-up data on household characteristics and net status were collected using structured, pretested and interviewer-administered questionnaires. The questionnaires were prepared in English and then translated into the local language, Afan Oromo. The USAIDsupported Malaria Consortium NetWorks training guideline was used to train data collectors for 2 days on the LLIN hole assessment technique [35]. All data collectors were diploma graduate personnel. Three teams of data collectors, each of which was comprised of three members (one supervisor and two data collectors in each 
team), were involved in data collection. During data collection, heads of households or competent family members (age $\geq 18$ years) were interviewed about the status of their LLINs. If LLINs were not found or used for other purposes, the respondents were asked why and how nets were lost or damaged or used for other purposes. If the visited house was closed or no competent (age $\geq 18$ years) respondent was present, the house was revisited at least three times within a week. If the house was closed or no competent respondent was present after three visits, LLINs were considered lost to follow-up.

\section{Definition and follow up of outcome variables Attrition}

Attrition was defined as the proportion of LLINs no longer in household use [34]. Attrition of LLINs was categorized as "attrition for known outcome" and "attrition for unknown outcome”.

\section{Attrition for known outcome}

Net lost from household due to discarding, destruction, or used for other purposes.

\section{Attrition for unknown outcome}

Net lost from household due to being given away for others to use, used in different location, stolen, sold or lost to follow up (due to family moving to other location or not at home).

\section{Physical integrity}

The physical integrity of the LLINs was defined considering the number, size, and location of holes to estimate the protection ability of the net against mosquito bites. For nets presented and used for sleeping during data collection, inspections were carried out for the presence, type, location, and size of holes. A rectangular metal frame with a size of $165 \mathrm{~cm}$ width, $185 \mathrm{~cm}$ length, and $160 \mathrm{~cm}$ height was used to hang and inspect each net for holes. Hole categories recommended by the WHO were used to determine hole size [20]. Hole-size categories were defined as follows: hole size 1, 0.5-2 cm (smaller than a thumb); hole size 2, 2-10 cm (larger than a thumb, but smaller than a fist); hole size 3, 10-25 cm (larger than a fist, but smaller than a head); and hole size 4, larger than $25 \mathrm{~cm}$ (larger than a head). Holes smaller than $0.5 \mathrm{~cm}$ were not counted. Moreover, the causes of holes were identified and evidence of repair was recorded. The proportional hole index (pHI) was used to group LLINs into serviceable or torn categories. The $\mathrm{pHI}$ for each LLIN was calculated by weighting each hole by its size (size 1-4) and totaling up the weighted number of holes as described elsewhere [34]. The LLINs with holes were categorized into one of the following groups: pHI 0-64, "good condition": no reduction of efficacy compared to an undamaged net; pHI 65-642, "acceptable condition": effectiveness somewhat reduced, but still provides significantly more protection than no net at all; and $\mathrm{pHI} \geq 643$, "torn" or poor physical integrity condition: the protective efficacy is in serious doubt, and the LLIN should be replaced as quickly as possible. The number of combined LLINs in "good" and "acceptable" condition represented the number of LLINs in "serviceable" condition or in good physical integrity condition [34].

\section{Functional survivorship}

Functional survival was defined as the proportion of LLINs in serviceable ("good" + "acceptable") condition at a given time point after LLIN distribution. Both attrition with known outcome and LLINs in serviceable or torn conditions were used to evaluate functional survival [36].

\section{Bio-efficacy}

The ability of a net to incapacitate or kill susceptible Anopheles mosquitoes after contact with the insecticide on the LLIN. For the bio-efficacy test, five samples from each LLIN measuring $30 \mathrm{~cm} \times 30 \mathrm{~cm}$ were cut according to the guideline [34]. Each piece of the net section was labelled with a unique identification number by combining the household number and a sample location. The samples were then wrapped in a foil and placed in a black plastic bag for storage until the test. In the laboratory, 10 susceptible, 2- to 5-day-old, non-blood fed female $A n$. arabiensis mosquitoes were exposed for $3 \mathrm{~min}$ on each piece of sample according to the WHO cone bioassay test procedure [12]. Control tests were carried out each day immediately before and after exposure of mosquitoes to experimental LLINs. The LLINs fulfilling the criteria of $\geq 95 \%$ KD or $\geq 80 \%$ mortality using susceptible Anopheles mosquitoes were considered effective [34].

\section{Statistical analysis}

Data were entered into and analysed by IBM SPSS version 20.0. For non-normally distributed continuous variables, medians and the interquartile range (IQR) were calculated. The dependent variables of the study were attrition, physical integrity, functional survival, and the bio-efficacy of LLINs. The exposure variables were gender and educational status of the head of the household, family size, wealth status, the presence of open eave gaps in the house, type of bed, status of net use, status of net washing, hygienic condition of the LLIN, presence of rodents or cats in the household and distance of household from vector breeding sites.

The household wealth index was calculated using principal component analysis (PCA) [37, 38]. Fourteen household assets were used in the calculations, 
including presence of electricity, ownership of television, radio, mobile telephone, chair, table, bed, bicycle, land, separate kitchen from living house, animal and animal cart, and types of roof and walls. A wealth index was constructed from the first principal component for each household, and then categorized into three relative measures of socioeconomic class (poor, middle, and rich). The Kaiser-Meyer-Olkin (KMO) measure of sample adequacy was 0.77 . The total variance explained by the first principal component was $23.8 \%$, with a corresponding Eigen value of 3.33 .

The attrition rate of the LLINs was calculated as the number of LLINs lost with known outcome of attrition or torn, divided by all LLINs enrolled at baseline. However, the LLINs lost with unknown outcome of attrition were excluded from the denominator. The physical integrity of the net was determined using two measurements. The first measurement was the proportion of LLINs with a hole size $0.5 \mathrm{~cm}$ or larger divided by the total number of coded LLINs found and assessed in the surveyed households. The second measurement was the proportion of torn nets divided by all nets assessed for holes. To estimate the proportion of functionally surviving LLINs, the nets in "good" and "acceptable" condition were used as a numerator, and all nets present in surveyed households and nets lost due to "known outcome of attrition" + torn were used as a denominator. The proportion of functionally surviving nets was compared against reference survival curves provided by the WHO [36]. A Kaplan-Meier survival analysis was used to estimate the median survival time of functionally surviving LLINs. The proportion of LLINs with effective bio-efficacy was calculated as the number of effective LLINs $(\geq 95 \%$ KD or $\geq 80 \%$ mortality) divided by the total number of LLINs tested. The LLINs were considered effective against malariatransmitting Anopheles mosquitoes if at least $80 \%$ of the sampled LLINs fulfilled the criteria of $\geq 95 \% \mathrm{KD}$ or $\geq 80 \%$ mortality after at least 20 washes and 3 years of use [34].

To investigate the predictors of physical integrity and functional survival of LLINs, a proportional Cox regression model was fitted to the dataset. The failure endpoint for physical integrity was defined as an LLIN in torn condition. And, for functional survival, the failure endpoint was either the LLIN having the known outcome of attrition or being in torn condition. The LLINs in the unknown outcome of attrition category were censored at the time of net loss. Variables having a $P$ value $<0.25$ in bivariate analysis were included in the multivariate analysis to identify independent predictors. A P value $<0.05$ was considered statistically significant.

\section{Ethical considerations}

Ethical clearance was obtained from the Ethiopian Ministry of Science and Technology (Ref: 3.10/446/06), Institutional Review Board of the College of Health Sciences at Addis Ababa University and the Regional Committee for Medical and Health Research Ethics, Western Norway (Ref: 2013/986/REK vest). Also, permission letters were obtained from the Oromia Regional Health Bureau, East Shewa Zonal Health Department, and Adami Tullu District Health Office. Information about the study objectives, procedures and benefits were clearly explained to the study participants. Written consent was not obtained because the majority of the study participants could not read or write [32]. Therefore, verbal informed consent was obtained from study participants during data collection.

\section{Results}

Characteristics of study households

A total of 659 households were included in this study. The majority of heads of households were male (407; $62 \%)$ and illiterate $(369 ; 57 \%)$. About 331 (50\%) of study households had a family size of more than five individuals. Approximately one-third of households (202; 31\%) lived within $1 \mathrm{~km}$ from a potential vector breeding site (Table 1).

\begin{tabular}{|c|c|}
\hline Variable & n (\%) \\
\hline \multicolumn{2}{|c|}{ Gender of head of household } \\
\hline Male & $407(61.8)$ \\
\hline Female & $252(38.2)$ \\
\hline \multicolumn{2}{|c|}{ Educational status of head of household $(n=647)$} \\
\hline |lliterate & $369(57.0)$ \\
\hline Read and write & $59(9.1)$ \\
\hline Primary & $162(25.0)$ \\
\hline Secondary and above & $57(8.8)$ \\
\hline \multicolumn{2}{|l|}{ Wealth status $(n=622)$} \\
\hline Poor & $230(37.0)$ \\
\hline Middle & $198(31.8)$ \\
\hline Rich & $194(31.2)$ \\
\hline \multicolumn{2}{|l|}{ Family size } \\
\hline$\leq 5$ & $328(49.8)$ \\
\hline$>5$ & $331(50.2)$ \\
\hline \multicolumn{2}{|c|}{ House with open eave gap $(n=615)$} \\
\hline Yes & $99(16.1)$ \\
\hline No & $516(83.9)$ \\
\hline \multicolumn{2}{|c|}{ Distance from mosquito breeding site (km) } \\
\hline$\leq 1$ & $202(30.7)$ \\
\hline$>1$ & $457(69.3)$ \\
\hline
\end{tabular}


Table 2 Reasons for loss of long-lasting insecticide nets over a 2-year follow-up period in Ethiopia

\begin{tabular}{|c|c|c|c|c|c|}
\hline Reason for LLIN loss & $\begin{array}{l}6 \text { months } \\
\mathrm{n}(\%)\end{array}$ & $\begin{array}{l}12 \text { months } \\
\text { n (\%) }\end{array}$ & $\begin{array}{l}18 \text { months } \\
\mathrm{n}(\%)\end{array}$ & $\begin{array}{l}24 \text { months } \\
\text { n (\%) }\end{array}$ & $\begin{array}{l}\text { Total } \\
\text { n (\%) }\end{array}$ \\
\hline \multicolumn{6}{|c|}{ Known outcome of attrition or torn } \\
\hline Thrown away & $102(40.0)$ & $301(71.8)$ & $173(74.9)$ & $62(70.5)$ & $638(64.2)$ \\
\hline Used for something else & $30(11.8)$ & $58(13.8)$ & $28(12.1)$ & $22(25.0)$ & $138(13.9)$ \\
\hline Torn $(\mathrm{pHI}>643)$ & $123(48.2)$ & $60(14.3)$ & $30(13.0)$ & $4(4.5)$ & $217(21.9)$ \\
\hline Total & $161(100)$ & $331.1(100)$ & $149(100)$ & $12(100)$ & $993(100)$ \\
\hline \multicolumn{6}{|c|}{ Unknown outcome of attrition } \\
\hline Given away & $260(76.7)$ & $44(45.8)$ & $11(20.0)$ & $1(12.5)$ & $316(63.5)$ \\
\hline Lost to follow-up a & $52(15.3)$ & $43(44.8)$ & $26(47.2)$ & $5(62.5)$ & $126(25.3)$ \\
\hline Stolen & $8(2.4)$ & $9(9.4)$ & $3(5.5)$ & $2(25.0)$ & $22(4.4)$ \\
\hline Unknown reasons & $6(1.8)$ & $0(0.0)$ & $1(1.8)$ & $0(0.0)$ & $7(1.4)$ \\
\hline Other $^{b}$ & $13(3.8)$ & $0(0.0)$ & $14(25.5)$ & $0(0.0)$ & $27(5.4)$ \\
\hline Total & $461(100)$ & $558.2(100)$ & $153(100)$ & (168) (100) & $498(100)$ \\
\hline
\end{tabular}

LLIN long-lasting insecticidal net

a Family moved to other location, family not at home, refusal to participate

b Sold or destroyed by fire

\section{Enrollment of LLINs and study completion}

At the start of the study, 1532 LLINs were included in the study for attrition and functional survival assessment. Out of this number, 1061 at 6 months $\left(\mathrm{T}_{6}\right), 517$ at 12 months $\left(\mathrm{T}_{12}\right), 198$ at 18 months $\left(\mathrm{T}_{18}\right)$, and 56 at 24 months $\left(\mathrm{T}_{24}\right)$ were available for examination.

\section{Attrition}

A total of 1491 LLINs were lost during the 2-year followup period. Among the lost LLINs, 993 (67\%) were lost due to a known outcome or torn, and 498 (33\%) were lost due to an unknown outcome (Table 2). The attrition for known outcomes or torn of LLINs increased more rapidly over time (Fig. 2). The overall attrition for known outcomes or torn from the beginning to the end of the study was 96\% (95\% CI 94.7-97.1; $\mathrm{n}=993 ; \mathrm{N}=1034)$. The reasons for this attrition were as follows: being

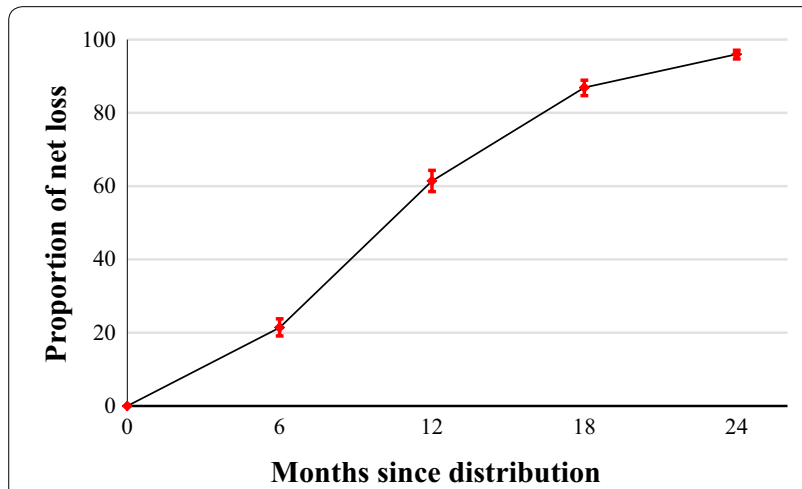

Fig. 2 Proportion of lost LLINs due to known reasons of attrition or torn over a 2 -year-period. The error bars indicate the $95 \%$ confidence interval thrown away because of damage (638; 64.2\%), torn (217; $21.9 \%)$, and being used for other purposes (138; $13.9 \%$; Table 2).

\section{Physical integrity}

The number of eligible LLINs, and those included for physical integrity evaluation at 6 months, as well as the number of LLINs found in the households during follow up are summarized in Fig. 3. The proportion of LLINs with a hole corresponding to the size categories $1-4$ was $35.8 \%$ (298 of 833 ) after 6 months. This proportion increased to $79.5 \%$ (31 of 39) after 24 months of follow up. When the locations of holes were considered, the mean number of holes of any size was found to be higher in the lower half of the LLIN compared with the upper half or the roof. The median $\mathrm{pHI}$ increased from month 6 to 18, whereas the pHI decreased slightly at 24 months (Table 3).

The proportions of LLINs in the "good" and "acceptable" categories decreased with age, whereas LLINs in the "torn" category increased with age. The proportion of torn LLINs increased from $14.8 \%$ (123 of 833) to $23.1 \%$ (9 of 39) between 6 and 24 months. Only 39 LLINs were identified during follow up visits. Among these, only one LLIN was torn at 6 months, and the number of torn LLINs increased to nine (23.1\%) after 24 months (Table 4).

\section{Predictors of physical integrity of LLINs}

A bivariate proportional Cox regression analysis showed that using the LLIN during the night before the day of the survey, having a clean LLIN, and the presence of a cat in the house were all associated with 


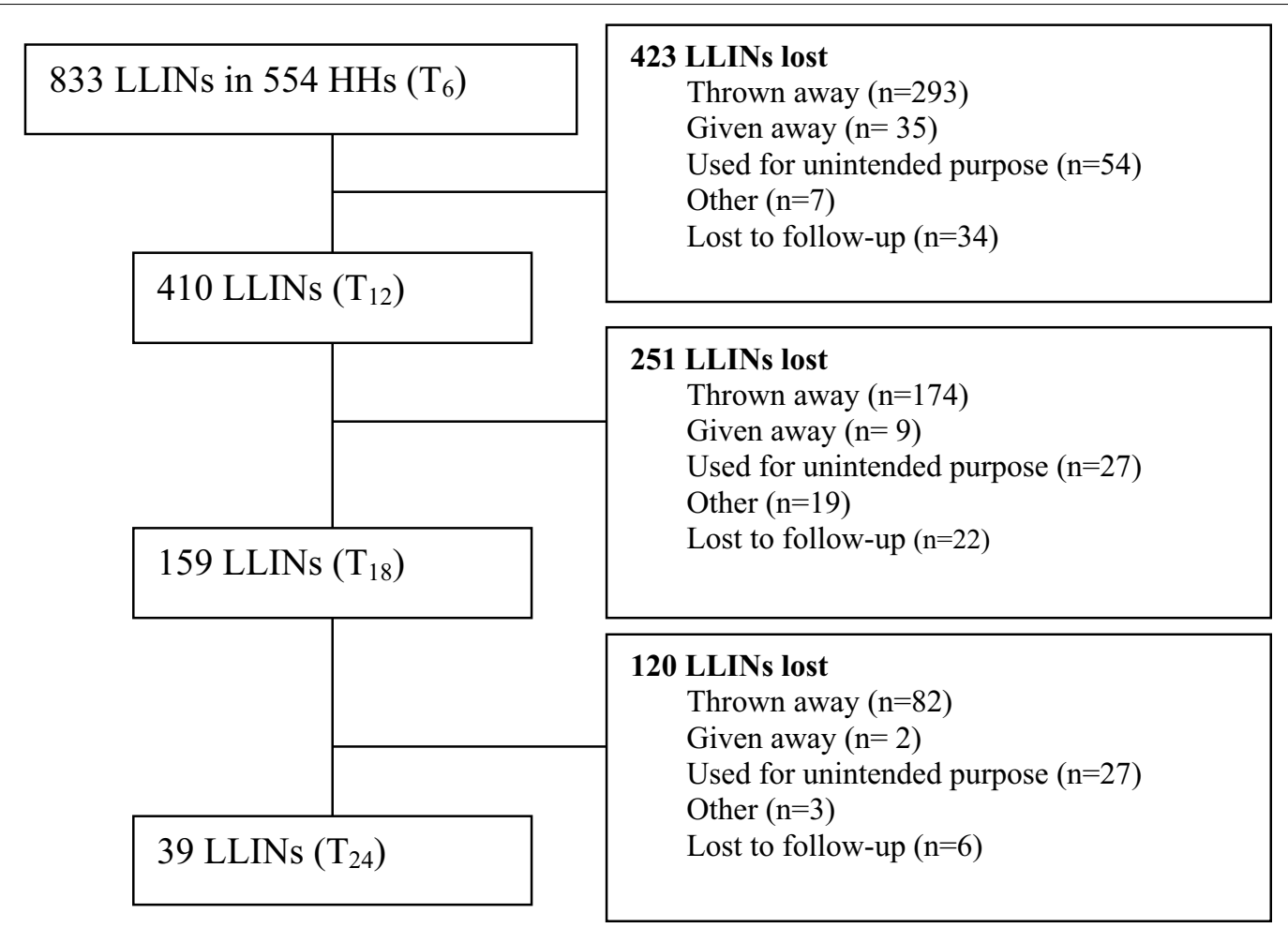

Fig. 3 Flow diagram shows the number of LLINs enrolled at 6 months in south-central Ethiopia

Table 3 Proportion of long-lasting insecticide nets with holes, mean number of holes by location, and proportional hole index over time in Ethiopia

\begin{tabular}{|c|c|c|c|c|}
\hline Characteristic & $\begin{array}{l}6 \text { months } \\
\mathrm{N}=833\end{array}$ & $\begin{array}{l}12 \text { months } \\
\mathrm{N}=410\end{array}$ & $\begin{array}{l}18 \text { months } \\
N=159\end{array}$ & $\begin{array}{l}24 \text { months } \\
N=39\end{array}$ \\
\hline Holes (size categories 1-4), n (\%) & $298(35.8)$ & $204(49.8)$ & $107(67.3)$ & $31(79.5)$ \\
\hline \multicolumn{5}{|l|}{ Mean (SD) number of holes } \\
\hline Lower half segment of nets & $3.7(12.7)$ & $4.2(12.0)$ & $5.7(13.3)$ & $4.4(6.6)$ \\
\hline Upper half segment of nets & $2.1(8.1)$ & $2.2(4.6)$ & $3.7(8.1)$ & $5(9.2)$ \\
\hline Roof segment of nets & $1.2(4.9)$ & $1.8(4.6)$ & $2.6(4.5)$ & $3.3(3.8)$ \\
\hline GM pHI $(95 \% \mathrm{Cl})^{\mathrm{a}}$ & $216(167-279)$ & $251(195-323)$ & $316(234-427)$ & $211(117-378)$ \\
\hline Median pHI (IQR) ${ }^{\mathrm{a}}$ & $270(48-993)$ & $275(88-843)$ & $422(173-775)$ & $296(77-604)$ \\
\hline
\end{tabular}

$\mathrm{N}$ : The total number of LLINs available for evaluation at each data collection period

$\mathrm{CI}$ Confidence interval, IQR interquartile range, LLIN long-lasting insecticidal net, SD standard deviation, GM geometric mean, $\mathrm{pHI}$ proportional hole index

a Number of LLINs evaluated ( $\mathrm{n}=274$ at 6 months, $\mathrm{n}=170$ at 12 months, $\mathrm{n}=85$ at 18 months, and $\mathrm{n}=28$ at 24 months)

the good physical integrity of LLINs. The presence of rats in the house and a household location of more than $1 \mathrm{~km}$ from a mosquito breeding site were associated with poor physical integrity. Multivariate analysis indicated that using a LLIN during the previous night (adjusted hazard ratio $[\mathrm{HR}]=0.7 ; 95 \%$ CI $0.50-0.98$ ), having a clean LLIN (adjusted $\mathrm{HR}=0.4$; 95\% CI 0.30 0.60 ), and being in a household more than $1 \mathrm{~km}$ away from a mosquito breeding site (adjusted HR 1.8; 95\% CI 1.2-2.6) were independent predictors of the physical integrity of LLINs (Table 5). 
Table 4 Proportion of long-lasting insecticide nets in good, acceptable, or torn condition over time, as defined by the proportional hole index in Ethiopia

\begin{tabular}{|c|c|c|c|c|}
\hline Category defined by $\mathrm{pHI}$ & $\begin{array}{l}6 \text { months } \\
\mathrm{N}=833 \\
\mathrm{n}(\%)\end{array}$ & $\begin{array}{l}12 \text { months } \\
N=410 \\
n(\%)\end{array}$ & $\begin{array}{l}18 \text { months } \\
N=159 \\
n(\%)\end{array}$ & $\begin{array}{l}24 \text { months } \\
N=39 \\
n(\%)\end{array}$ \\
\hline Good (0-64) & $610(73.2)$ & $243(59.3)$ & $63(39.6)$ & $14(35.9)$ \\
\hline Acceptable (65-642) & $100(12.0)$ & $82(9.8)$ & $45(28.3)$ & $16(41.0)$ \\
\hline Torn $(>643)$ & $123(14.8)$ & $85(20.7)$ & $51(32.1)$ & $9(23.1)$ \\
\hline \multicolumn{5}{|c|}{ LLINs present at all follow-up visits, $n=39$} \\
\hline Good $(0-64)$ & $34(87.2)$ & $29(74.4)$ & 19 (48.7) & $14(35.9)$ \\
\hline Acceptable (65-642) & $4(10.3)$ & $6(15.4)$ & $13(33.3)$ & $16(41.0)$ \\
\hline Torn $(>643)$ & $1(2.6)$ & $4(10.3)$ & $7(17.9)$ & $9(23.1)$ \\
\hline
\end{tabular}

$\mathrm{N}$ : The total number of LLINs available for evaluation at each data collection period

LLIN long-lasting insecticidal net, $p H I$ proportional hole index

\section{Functional survival}

Observed functional survivals at different time points, compared with the reference NetCALC loss prediction curves, are shown in Fig. 4. Six months after distribution, 78.6\% (95\% CI 76.2-80.9) of the LLINs were functionally surviving. However, by month 24 , only $4 \%$ (95\% CI 2.9-5.4) had survived. The median (95\% CI) survival time (time since distribution, in which $50 \%$ of LLINs were in a serviceable condition) was $12(11.6-12.4)$ months. The observed functional survival was less than the 3-year serviceable model, being closer to the 1-year serviceable model (Fig. 4).

\section{Predictors of functional survivorship of LLINs}

A multivariate proportional Cox regression model showed that having a clean LLIN (adjusted HR $=0.8 ; 95 \%$ CI 0.6-0.9) was an independent predictor of longer functional survival, whereas the distance of a household $1 \mathrm{~km}$ from a mosquito breeding site (adjusted $\mathrm{HR}=1.3$; 95\% CI 1.1-1.6) was associated with the shorter functional survival of LLINs (Table 6).

\section{Bio-efficacy}

A total of 120 LLINs were tested using WHO cone bioassays over a 2-year period. The GM of 60 -min KD rates was greater than $90 \%$ in all four surveys. The GM of $24-\mathrm{h}$ mortality rates was below $80 \%$ in the second year with $76.6 \%(95 \%$ CI 71.0-82.6) at 12 months and 69.4\% (95\% CI 59.4-80.9) at 24 months (Table 7). Statistically significant differences were observed both in 60-min KD rates, and 24-h mortality rates between 12 and 18 months and between 12 and 24 months (Table 8 ).

The proportion of LLINs meeting the WHO pesticide evaluation scheme criteria at different time points is presented in Table 9. At 6 months, the proportion of LLINs meeting the criteria of effective bio-efficacy was $90 \%$ (95\% CI 72.5-96.8), and this proportion decreased to $80 \%$ (95\% CI 61.5-90.9) at 24 months. However, LLINs met the criteria of effective bio-efficacy in all study periods.

\section{Discussion}

Low functional survivorship of LLINs was observed in south-central Ethiopia. The data show that most LLINs survive for approximately 1 year. High attrition rates due to discarding and the poor physical integrity of LLINs were the major causes of low functional survivorship. The LLINs were found to be effective against malaria-transmitting mosquitoes and met the criteria of optimal effectiveness of bio-efficacy up to month 24.

Previous studies have used cross-sectional study designs to evaluate the durability of LLINs in Ethiopia $[17,21]$. Because of the design, these studies could not quantify attrition, functional survivorship, and changes in the physical integrity of LLINs over time. The current study has addressed these limitations. Unlike previous follow-up studies $[8,13,39]$, this study followed all LLINs in selected households to avoid selection bias and potential observer effects (Hawthorne effect) in which users might treat the net under observation differently than nets not under observation.

This study had some limitations. The prospective nature of the study may have influenced the user to keep their LLINs longer because they were being observed. However, because the attrition rate was much higher than expected, this potential limitation is less likely to have influenced the results. The functional survival time of LLINs may have been overestimated because LLINs could be lost at any time during the 6-month follow-up period. There may have also been recall bias, as people may not have correctly remembered what had happened to their LLINs over the previous 6 months. Furthermore, 
Table 5 Predictors of physical integrity of long-lasting insecticide nets over a 2-year follow-up period in Ethiopia

\begin{tabular}{|c|c|c|c|c|c|}
\hline Variables & $\begin{array}{l}\text { Net months } \\
\text { observation }\end{array}$ & $\begin{array}{l}\text { Number of torn } \\
\text { LLINs }\end{array}$ & IR/100 NMO (95\% Cl) & Crude HR $(95 \% \mathrm{Cl})$ & Adjusted HR (95\% Cl) \\
\hline \multicolumn{6}{|l|}{ Gender of head of household } \\
\hline Male & 5250 & 138 & $2.6(2.2-3.1)$ & 1.00 & 1.00 \\
\hline Female & 2958 & 60 & $2.0(1.5-2.5)$ & $0.8(0.6-1.0)$ & $0.8(0.6-1.3)$ \\
\hline \multicolumn{6}{|c|}{ Educational status of head of household $(n=816)$} \\
\hline Illiterate & 4458 & 108 & $2.4(2.0-2.9)$ & 1.0 & NA \\
\hline Read and write & 822 & 19 & $2.3(1.4-3.5)$ & $1.0(0.6-1.6)$ & \\
\hline Primary & 2010 & 44 & $2.2(1.5-2.8)$ & $0.9(0.6-1.3)$ & \\
\hline Secondary and above & 786 & 18 & $2.3(1.2-3.3)$ & $1.0(0.6-1.6)$ & \\
\hline \multicolumn{6}{|l|}{ Wealth status } \\
\hline Poor & 2826 & 77 & $2.7(2.1-3.3)$ & 1.0 & 1.0 \\
\hline Middle & 2640 & 62 & $2.3(1.8-2.9)$ & $0.9(0.6-1.2)$ & $1.0(0.7-1.5)$ \\
\hline Rich & 2742 & 59 & $2.2(1.6-2.7)$ & $0.8(0.6-1.1)$ & $0.9(0.6-1.4)$ \\
\hline \multicolumn{6}{|l|}{ Type of bed } \\
\hline Wooden bedframe & 3786 & 86 & $2.3(1.8-2.8)$ & 1.0 & NA \\
\hline Stick or iron bedframe & 924 & 23 & $2.5(1.5-3.5)$ & $1.1(0.7-1.8)$ & \\
\hline Mattress (with no bed frame) & 2340 & 56 & $2.4(1.8-3.0)$ & $1.1(0.8-1.5)$ & \\
\hline Mat & 1158 & 33 & $2.8(1.9-3.8)$ & $1.2(0.8-1.9)$ & \\
\hline \multicolumn{6}{|l|}{ LLIN hung up } \\
\hline No & 2358 & 64 & $2.7(2.0-3.4)$ & 1.0 & NA \\
\hline Yes & 5850 & 134 & $2.3(1.9-2.7)$ & $0.8(0.6-1.1)$ & \\
\hline \multicolumn{6}{|c|}{ House has open eave gap $(n=820)$} \\
\hline No & 6888 & 160 & $2.3(2.0-2.7)$ & 1.0 & 1.0 \\
\hline Yes & 1158 & 38 & $3.3(2.2-4.3)$ & $1.4(0.98-2.0)$ & $1.0(0.6-1.4)$ \\
\hline \multicolumn{6}{|l|}{ LLIN used last night } \\
\hline No & 2496 & 75 & $3.0(2.3-3.7)$ & 1.0 & 1.0 \\
\hline Yes & 5712 & 123 & $2.2(1.8-2.5)$ & $0.7(0.5-0.96)^{*}$ & $0.7(0.5-0.96)^{*}$ \\
\hline \multicolumn{6}{|l|}{ LLIN ever washed } \\
\hline No & 4992 & 109 & $2.2(1.8-2.6)$ & 1.0 & 1.0 \\
\hline Yes & 3216 & 89 & $2.8(2.2-3.3)$ & $1.3(1.0-1.7)$ & $1.2(0.9-1.7)$ \\
\hline \multicolumn{6}{|l|}{ LLIN was clean } \\
\hline No & 4440 & 156 & $3.5(3.0-4.1)$ & 1.0 & 1.0 \\
\hline Yes & 3768 & 42 & $1.1(0.8-1.5)$ & $0.3(0.2-0.4)^{*}$ & $0.4(0.3-0.6)^{*}$ \\
\hline \multicolumn{6}{|c|}{ Rats present in the house $(n=658)$} \\
\hline No & 3900 & 73 & $1.9(1.4-2.3)$ & 1.0 & 1.0 \\
\hline Yes & 3186 & 82 & $2.6(2.0-3.1)$ & $1.4(1.02-1.92)^{*}$ & $1.1(0.8-1.5)$ \\
\hline \multicolumn{6}{|l|}{ Cat present in the house $(n=658)$} \\
\hline No & 3648 & 94 & $2.6(2.1-3.1)$ & 1.0 & 1.0 \\
\hline Yes & 3438 & 61 & $1.8(1.3-2.2)$ & $0.7(0.5-0.9)^{*}$ & $0.8(0.5-1.1)$ \\
\hline \multicolumn{6}{|c|}{ Distance from mosquito breeding site $(n=833)(\mathrm{km})$} \\
\hline$\leq 1$ & 2844 & 51 & $1.8(1.3-2.3)$ & 1.0 & 1.0 \\
\hline$>1$ & 5364 & 147 & $2.7(2.3-3.1)$ & $1.5(1.1-2.1)^{*}$ & $1.8(1.2-2.6)^{*}$ \\
\hline \multicolumn{6}{|l|}{ Family size $(n=833)$} \\
\hline$\leq 5$ & 3594 & 90 & $2.5(2.0-3.0)$ & 1.0 & NA \\
\hline$>5$ & 4614 & 108 & $2.3(1.9-2.8)$ & $0.9(0.7-1.2)$ & \\
\hline
\end{tabular}




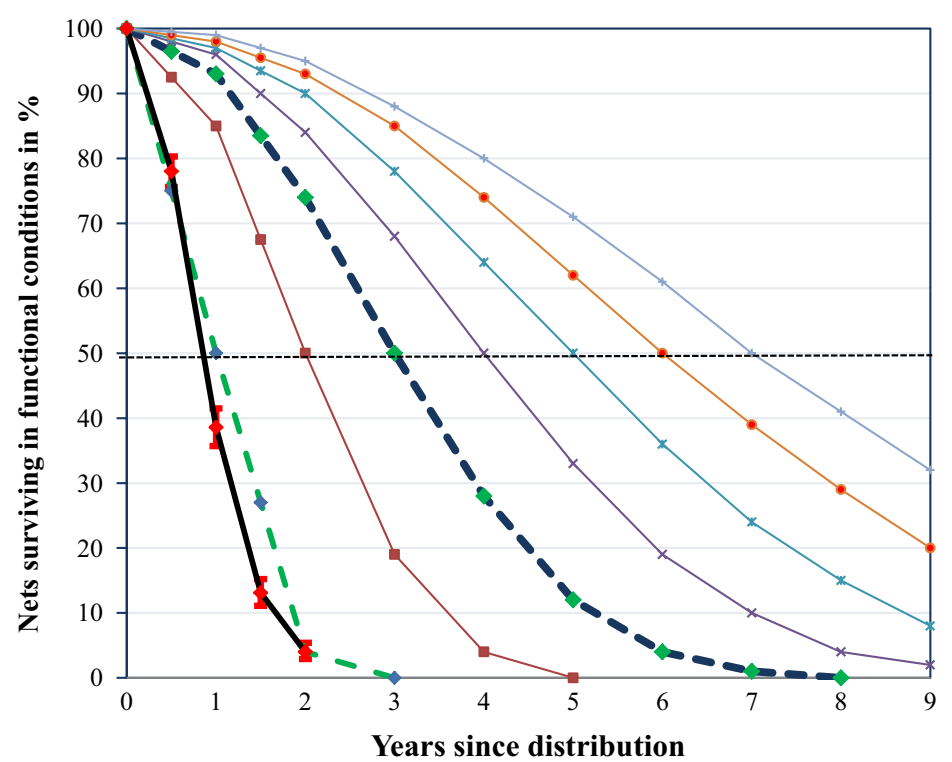

Hypothetical survival curves of defined median survival

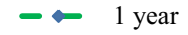

$\longrightarrow 2$ year

$-\infty-3$ year

$\longrightarrow 4$ year

$\div-5$ year

$\longrightarrow 6$ year

$\longrightarrow 7$ year

$\longrightarrow$ Current Study

Years since distribution

Fig. 4 Functional survival of LLINs in south-central Ethiopia. The error bars indicate the $95 \%$ confidence interval

it was difficult to trace the reason for LLIN loss when more than one LLIN was lost within the same household.

As expected, the physical integrity of LLINs deteriorated over time. The proportion of LLINs with a hole size $0.5 \mathrm{~cm}$ and larger (36-80\% of LLINs) between 6-24 months was comparable to other studies in Ethiopia (54.5-85.5\%) between 6-20 months [17], and in Zambia (60.2-87.2\%) between 12-24 months [8]. The observed high number of holes in the lower half of the nets was also consistent with the findings of previous studies [8, $10,17,40]$. The previous study reported that using nets over a reed mat was significantly associated with larger holes in the lower half of the nets [8].

Using the LLIN the night before the survey was associated with the good physical integrity of LLINs. Net use and having good physical integrity might have a bi-directional association. The users might keep their in-use nets from physical damage. Conversely, the users might prefer to use intact nets more than damaged nets. Having a clean LLIN was another predictor of the good physical integrity of LLINs in this study. This finding also might be due to the tendency of users to keep an intact net clean for prolonged use compared with damaged nets. Moreover, the presence of kitchens inside the house or using firewood as a cooking fuel could make the nets dirty [13]. Thus, dirty nets may be frequently washed, and could lose their physical integrity. Proximity to a mosquito breeding site was a significant predictor of physical integrity. The LLINs in households that lived more than $1 \mathrm{~km}$ away from potential vector breeding sites were more likely to be damaged than households located within a $1 \mathrm{~km}$ radius. This finding could be explained by nets being less valued in areas with a lower perceived risk of mosquito bites and malaria infection. Evidence from the qualitative study showed the tendency of owners far from potential mosquito breeding sites to misuse nets [14].

Six months after distribution, the functional survivorship of LLINs was $78.6 \%$. This percentage is lower than that reported in a study in Benin (93\%) [13]. One potential explanation for this difference is that all LLINs presented in the household, including torn nets, were considered to have survived in the Benin study. Moreover, the percentage of surviving nets in this study is lower than the NetCALC model 3-year serviceable prediction value of $96.5 \%$ [41]. After 12 months, net survivorship further decreased to $38.6 \%$, lower than the $72 \%$ reported from Benin [13] and $90.4 \%$ in Zambia [8]. In our area, the greatest loss (40\%) occurred during the 6- to 12-month period after LLIN distribution, and is probably related to the unusually dry and warmer weather that followed the El Nino in 2015 [30]. Moreover, the marked decline in the incidence of malaria in the study area (only $37 \%$ of predistribution incidence) [42] could have indirectly affected the survivorship of LLINs by decreasing the perceived risk of malaria infection. After 24 months, the functional survival was only $4 \%$, which is substantially lower than the expected $75 \%$ by the NetCALC 3-year serviceable prediction model [41]. In general, the functional survival of LLINs in the current study is comparable to a 1-year serviceable prediction model, in which $4 \%$ of LLINs are predicted to survive after 2 years $[36,41]$. 
Table 6 Predictors of functional survival of long-lasting insecticidal nets over a 2-year follow-up period in Ethiopia

\begin{tabular}{|c|c|c|c|c|c|}
\hline Variable & $\begin{array}{l}\text { Net month } \\
\text { observation }\end{array}$ & $\begin{array}{l}\text { Number of lost } \\
\text { LLINs }\end{array}$ & IR/100 NMO (95\% Cl) & Crude HR $(95 \% \mathrm{Cl})$ & $\begin{array}{l}\text { Adjusted HR (95\% } \\
\text { Cl) }\end{array}$ \\
\hline \multicolumn{6}{|c|}{ Gender of head of household $(n=1193)$} \\
\hline Male & 9930 & 660 & $6.6(6.1-7.2)$ & 1.0 & \multirow[t]{2}{*}{ NA } \\
\hline Female & 5262 & 333 & $6.3(5.6-7.0)$ & $1.0(0.8-1.1)$ & \\
\hline \multicolumn{6}{|c|}{ Educational status of head of household $(n=1170)$} \\
\hline Illiterate & 8472 & 567 & $6.7(6.1-7.2)$ & 1.0 & 1.0 \\
\hline Read and write & 1554 & 99 & $6.4(5.1-7.6)$ & $0.9(0.7-1.1)$ & $0.9(0.7-1.3)$ \\
\hline Primary & 3606 & 227 & $6.3(5.5-7.1)$ & $0.9(0.8-1.1)$ & $1.0(0.9-1.3)$ \\
\hline Secondary and above & 1308 & 79 & $6.0(4.7-7.4)$ & $0.9(0.7-1.1)$ & $0.9(0.7-1.2)$ \\
\hline \multicolumn{6}{|l|}{ Wealth status $(n=1184)$} \\
\hline Poor & 5244 & 363 & $6.9(6.2-7.6)$ & 1.0 & 1.0 \\
\hline Middle & 4872 & 312 & $6.4(5.7-7.1)$ & $0.9(0.8-1.0)$ & $1.0(0.8-1.2)$ \\
\hline Rich & 4968 & 312 & $6.3(5.6-7.0)$ & $0.9(0.8-1.0)$ & $0.9(0.8-1.2)$ \\
\hline \multicolumn{6}{|l|}{ Open eave $(n=1176)$} \\
\hline No & 12,480 & 819 & $6.6(6.1-7.0)$ & 1.0 & \multirow[t]{2}{*}{ NA } \\
\hline Yes & 2460 & 166 & $6.7(5.7-7.8)$ & $1.0(0.9-1.2)$ & \\
\hline \multicolumn{6}{|c|}{ LLIN used last night $(n=833)$} \\
\hline No & 3468 & 237 & $6.8(6.0-7.7)$ & 1.0 & 1.0 \\
\hline Yes & 7746 & 459 & $5.9(5.4-6.5)$ & $0.8(0.7-0.97)^{*}$ & $1.0(0.7-1.0)$ \\
\hline \multicolumn{6}{|c|}{ LLIN ever washed $(n=833)$} \\
\hline No & 6876 & 423 & $6.2(5.6-6.7)$ & 1.0 & \\
\hline Yes & 4338 & 273 & $6.3(5.5-7.0)$ & $1.0(0.9-1.2)$ & NA \\
\hline \multicolumn{6}{|l|}{ LLIN was clean $(n=833)$} \\
\hline No & 6012 & 418 & $7.0(6.3-7.6)$ & 1.0 & 1.0 \\
\hline Yes & 5202 & 278 & $5.3(4.7-6.0)$ & $0.7(0.6-0.8)^{*}$ & $0.8(0.6-0.9)^{*}$ \\
\hline \multicolumn{6}{|c|}{ Rats present in the house $(n=858)$} \\
\hline No & 6462 & 382 & $5.9(5.3-6.5)$ & 1.0 & 1.0 \\
\hline Yes & 5316 & 334 & $6.3(5.6-7.0)$ & $1.1(1.0-1.3)$ & $1.1(0.9-1.3)$ \\
\hline \multicolumn{6}{|c|}{ Cat present in the house $(n=858)$} \\
\hline No & 6102 & 378 & $6.2(5.6-6.8)$ & 1.0 & \\
\hline Yes & 5676 & 338 & $6.0(5.3-6.6)$ & $1.0(0.8-1.1)$ & NA \\
\hline \multicolumn{6}{|c|}{ Distance from mosquito breeding site $(n=1193)(\mathrm{km})$} \\
\hline$\leq 1$ & 5058 & 311 & $6.1(5.5-6.8)$ & 1.0 & 1.0 \\
\hline$>1$ & 10,134 & 682 & $6.7(6.2-7.2)$ & $1.2(1.1-1.3)^{*}$ & $1.3(1.1-1.6)^{*}$ \\
\hline \multicolumn{6}{|c|}{ Household population size $(n=1193)$} \\
\hline$\leq 5$ & 6264 & 392 & $6.3(5.6-6.9)$ & 1.0 & 1.0 \\
\hline$>5$ & 8928 & 601 & $6.7(6.2-7.3)$ & $1.1(1.0-1.3)$ & $1.1(0.9-1.3)$ \\
\hline
\end{tabular}

NMO net month observation, IR incidence rate, LLIN long-lasting insecticidal net, $H R$ hazard ratio, NA not applicable when $\mathrm{P}<0.25,{ }^{*}$ statistically significant at $\mathrm{P}<0.05$

Table 7 Geometric means of 1-h knockdown and 24-h mortality of mosquitoes in Ethiopia

\begin{tabular}{llll}
\hline $\begin{array}{l}\text { Survey } \\
\text { (months) }\end{array}$ & $\begin{array}{l}\text { Number } \\
\text { of LLINs }\end{array}$ & $\begin{array}{l}\mathbf{6 0 - m i n} \text { KD } \\
\text { GM }(\mathbf{9 5} \% \mathbf{C I})\end{array}$ & $\begin{array}{l}\mathbf{2 4 - h} \text { mortality } \\
\text { GM (95\% CI) }\end{array}$ \\
\hline 6 & 30 & $94.1(87.1-100)$ & $81.1(67.7-97.0)$ \\
12 & 30 & $99.9(99.7-100)$ & $89.5(87.2-91.8)$ \\
18 & 30 & $93.9(90.0-98.1)$ & $76.6(71.0-82.6)$ \\
24 & 30 & $94.1(91.3-97.1)$ & $69.4(59.4-80.9)$ \\
\hline
\end{tabular}

$\mathrm{Cl}$ confidence interval, GM geometric mean, KD knockdown, LLIN long-lasting insecticidal net
In addition to the unexpected weather conditions and a decline in the incidence of malaria, the behaviour of the net users could play a role in high attrition rate and low functional survivorship. A qualitative study done on the same households as our study showed that many informants believed that the LLINs would not serve more than 1 year. The users claimed the LLINs could lose their insecticidal effect after 6 months by mentioning that the nets "stopped killing bugs." Washing LLINs several times was also believed to cause a loss of insecticides [14]. As 


\begin{tabular}{llll}
$\begin{array}{l}\text { Table } 8 \\
\text { of knockdowns and mortality of }\end{array}$ & $\begin{array}{c}\text { differences } \\
\text { mosquitoes in Ethiopia }\end{array}$ \\
\hline Variable & Between months & $\begin{array}{c}\text { Mean difference } \\
\text { (95\% CI) }\end{array}$ & P value \\
\hline Knockdown & 12 and 18 & $5.4(0.4-10.4)$ & 0.029 \\
& 12 and 24 & $5.5(1.7-9.3)$ & 0.002 \\
Mortality & 12 and 18 & $11.7(4.1-19.2)$ & 0.001 \\
& 12 and 24 & $15.4(3.1-27.8)$ & 0.008 \\
\hline
\end{tabular}

Cl confidence interval

a One-way ANOVA assuming unequal variance used

Table 9 Proportion of long-lasting insecticidal nets meeting WHO pesticide evaluation scheme criteria effective (1-h knockdown $\geq 95 \%$ or $24-h$ mortality $\geq 80 \%$ ) in Ethiopia

\begin{tabular}{lll}
\hline Age of LLINs (months) & Number evaluated & $\mathbf{n}(\%), \mathbf{9 5 \%} \mathbf{C l}$ \\
\hline 6 & 30 & $27(90.0), 72.5-96.8$ \\
12 & 30 & $30(100)$ \\
18 & 30 & $25(83.3), 65.1-93.1$ \\
24 & 30 & $24(80.0), 61.5-90.9$ \\
\hline
\end{tabular}

Cl confidence interval, LLINs long-lasting insecticidal nets, WHO World Health Organization

explored by this qualitative study, after 1 year most of the LLINs were misused. However, this finding was not supported by the current study, as $64.2 \%$ of reported net loss was due to disposal. There could be a possible social desirability bias, because people did not report the misuse of LLINs in the current study. There is also a possibility that the LLINs were used for agricultural purposes, such as grain storage and transportation from the field, as well as the separation of grains from their chaffs, before being discarded as explored by the qualitative study [14]. Moreover, a low level of knowledge and a low positive perception towards net care and repair in Ethiopia may have also played a role in the observed high attrition, poor physical integrity and lower functional survival of LLINs [15].

In this study, having a clean LLIN was found to be associated with a longer functional survival time. This could be due to the behaviour of the owners, who would like to use LLINs for a prolonged time and thus keep the nets clean. A result from a qualitative study showed that nets become dirty from excessive smoke from indoor cooking stoves or fires, which leads the users to discard the nets prematurely or misuse them [14]. The LLINs in households living more than $1 \mathrm{~km}$ from potential vector breeding sites were less likely to survive. This could be related to a higher perceived risk of mosquito bites and malaria infection among net owners living closer to a vector breeding site $[43,44]$. In this study, neither using the net the night before the survey nor having ever washed the net was associated with functional survival of LLINs. However, a previous study observed an association between using the net the night before the survey and a longer survival time, and an association between having ever washed the net and a shorter survival time [8].

Previous studies have reported that the bio-efficacy of the LLIN is correlated with the concentration of the insecticide $[8,21]$. In the current study, PermaNet ${ }^{\circledR} 2.0$ LLINs met the WHO pesticide evaluation scheme criteria of bio-efficacy (at least $80 \%$ of the sampled LLINs effective in a WHO cone test) after 24 months [34], which was in agreement with other similar studies [21,39] and higher than a result reported by Tan et al. [8].

In general, our results suggested that the survivorship of LLINs after 2 years was low compared with the prediction of the NetCALC model (4\% vs 75\%). This finding raises a serious concern about the programmatic assumption of the 3-year LLIN replacement cycle. Therefore, we suggest that nationally representative LLIN durability studies should be conducted to estimate the correct LLIN replacement cycle. Meanwhile, national malaria control programs should closely work with manufacturers to develop stronger and more durable LLIN products. Moreover, strengthening the behaviour change communication messages on net care and repair, as well as the proper use of LLINs, may help to improve the durability of LLINs.

\section{Conclusions}

The study results suggested that the serviceable time of LLINs is 1 year, as a "3-year" serviceable assumption was unrealistic in this study community. Consequently, stronger and more efficient LLINs need to be developed for conditions similar to those studied here. After all, many parts of Ethiopia exhibit conditions similar to those at this study site. Because this study was conducted on one brand of LLIN and in one area only, the findings may not be extrapolated to other brands and people living in different topographic and socioeconomic settings. Therefore, more research still needs to be conducted to generalize the findings to the country level.

\section{Abbreviations \\ AHR: adjusted hazard ratio; Cl: confidence interval; GM: geometric mean; KD: knockdown; IQR: interquartile range; IRS: indoor residual spray; LLIN: long-lasting insecticidal net; MD: mean difference; PCA: principal component analysis; pHI: proportional hole index; WHO: World Health Organization.}

\section{Authors' contributions}

TS, EL, WD, MB, HJO, and BL conceived and designed the study, and designed data collection tools. TS, EL, and TG facilitated the data collection and supervised the field work. TS and MB conducted the bio-efficacy test. TS and BL analysed and interpreted the data and drafted the manuscript. All authors 
reviewed and edited the draft manuscript. All authors read and approved the final manuscript.

\section{Author details}

${ }^{1}$ School of Public and Environmental Health, Hawassa University, Hawassa, Ethiopia. ${ }^{2}$ Department of Preventive Medicine, School of Public Health, College of Health Sciences, Addis Ababa University, Addis Ababa, Ethiopia. ${ }^{3}$ Aklilu Lemma Institute of Pathobiology, Addis Ababa University, Addis Ababa, Ethiopia. ${ }^{4}$ Norwegian University of Life Sciences, Ås, Norway. ${ }^{5}$ Centre for International Health, University of Bergen, Bergen, Norway.

\section{Acknowledgements}

The authors would like to gratefully acknowledge all the study participants who took part in this study. We would like to thank the data collectors, supervisors, and the Adami Tullu district health office, all of whom facilitated the data collection process. We are also grateful for Hawassa University, Addis Ababa University, and the University of Bergen for their support.

\section{Competing interests}

The authors declare that they have no competing interests.

\section{Availability of data and materials}

The datasets used and analysed during the current study are available from the corresponding author on reasonable request.

\section{Consent for publication}

Not applicable.

\section{Ethics approval and consent to participation}

This study was approved by the Ethiopian Ministry of Science and Technology (Ref: 3.10/446/06) and the Regional Committee for Medical and Health Research Ethics, Western Norway (Ref: 2013/986/REK vest)

\section{Funding}

This study received financial support from the Research Council of Norway through the GLOBVAC programme (Project Number: 220554).

\section{Publisher's Note}

Springer Nature remains neutral with regard to jurisdictional claims in published maps and institutional affiliations.

Received: 26 May 2018 Accepted: 14 June 2018

Published online: 20 June 2018

\section{References}

1. WHO, UNICEF. Achieving the malaria MDG target: reversing the incidence of malaria 2000-2015. Geneva: World Health Organization and the United Nations Children's Fund. 2015. http://www.who.int/malaria/publications/ atoz/9789241509442/en/. Accessed 12 Sept 2017.

2. Misganaw A, Melaku YA, Tessema GA, Deribew A, Deribe K, Abera SF, et al. National disability-adjusted life years (DALYS) for 257 diseases and injuries in Ethiopia, 1990-2015: findings from the global burden of disease study 2015. Popul Health Metr. 2017:15:28.

3. WHO. World malaria report. Geneva: World Health Organization; 2017.

4. WHO. Roll back malaria partnership global malaria action plan. Geneva: World Health Organization. 2008. http://archiverbm.rollbackmalaria.org/ gmap/gmap.pdf. Accessed 20 Oct 2015.

5. WHO. World malaria report. Geneva: World Health Organization; 2015.

6. WHO. Achieving universal coverage with long-lasting insecticidal nets in malaria control. Geneva: World Health Organization. 2014. http:// www.who.int/malaria/publications/atoz/who_recommendation_cover age Ilin/en/. Accessed 2015 Apr 24.

7. Ethiopian Public Health Institute. Ethiopia National Malaria Indicator Survey 2015. Addis Ababa: Ethiopian Public Health Institute. 2016. https:// www.ephi.gov.et/images/pictures/download2009/MIS-2015-Final-Repor t-December-_2016.pdf. Accessed 8 June 2017.
8. Tan KR, Coleman J, Smith B, Hamainza B, Katebe-Sakala C, Kean C, et al. A longitudinal study of the durability of long-lasting insecticidal nets in Zambia. Malar J. 2016:15:106.

9. Massue DJ, Moore SJ, Mageni ZD, Moore JD, Bradley J, Pigeon O, et al. Durability of Olyset campaign nets distributed between 2009 and 2011 in eight districts of Tanzania. Malar J. 2016;15:176.

10. Morgan J, Abilio AP, do Rosario Pondja M, Marrenjo D, Luciano J, et al. Physical durability of two types of long-lasting insecticidal nets (LLINs) three years after a mass LLIN distribution campaign in Mozambique, 2008-2011. Am J Trop Med Hyg. 2015;92:286-93.

11. Kilian A, Koenker H, Obi E, Selby RA, Fotheringham M, Lynch M. Field durability of the same type of long-lasting insecticidal net varies between regions in Nigeria due to differences in household behaviour and living conditions. Malar J. 2015:14:123.

12. Hakizimana E, Cyubahiro B, Rukundo A, Kabayiza A, Mutabazi A, Beach R, et al. Monitoring long-lasting insecticidal net (LLIN) durability to validate net serviceable life assumptions, in Rwanda. Malar J. 2014;13:344.

13. Gnanguenon V, Azondekon R, Oke-Agbo F, Beach R, Akogbeto M Durability assessment results suggest a serviceable life of two, rather than three, years for the current long-lasting insecticidal (mosquito) net (LLIN) intervention in Benin. BMC Infect Dis. 2014;14:69.

14. Doda Z, Solomon T, Loha E, Gari T, Lindtjørn B. A qualitative study of use of long-lasting insecticidal nets (LLINs) for intended and unintended purposes in Adami Tullu, East Shewa Zone, Ethiopia. Malaria J. 2018;17:69.

15. Zewde A, Irish S, Woyessa A, Wuletaw Y, Nahusenay H, Abdelmenan S, et al. Knowledge and perception towards net care and repair practice in Ethiopia. Malar J. 2017:16:396.

16. WHO. Guidelines for laboratory and field testing of long-lasting insecticidal mosquito nets. Geneva: World Health Organization. 2005. http:// whqlibdoc.who.int/hq/2005/WHO_CDS_WHOPES_GCDPP_2005.11.pdf. Accessed 4 Dec 2014

17. Wills AB, Smith SC, Anshebo GY, Graves PM, Endeshaw T, Shargie EB, et al. Physical durability of PermaNet 2.0 long-lasting insecticidal nets over three to 32 months of use in Ethiopia. Malar J. 2013;12:242.

18. Mejia P, Teklehaimanot HD, Tesfaye $Y$, Teklehaimanot A. Physical condition of Olyset ${ }^{\circledR}$ nets after five years of utilization in rural western Kenya. Malar J. 2013;12:158.

19. Kilian A, Byamukama W, Pigeon O, Gimnig J, Atieli F, Koekemoer L, et al. Evidence for a useful life of more than three years for a polyester-based long-lasting insecticidal mosquito net in Western Uganda. Malar J. 2011;10:299.

20. WHO Guidelines for monitoring the durability of LLINs under operational conditions. Geneva: World Health Organization. 2011. http://apps.who. int/iris/bitstream/10665/44610/1/9789241501705_eng.pdf. Accessed 10 Sept 2014.

21. Anshebo GY, Graves PM, Smith SC, Wills AB, Damte M, Endeshaw T, et al. Estimation of insecticide persistence, biological activity and mosquito resistance to PermaNet ${ }^{\circledR} 2$ long-lasting insecticidal nets over three to 32 months of use in Ethiopia. Malar J. 2014:13:80.

22. Yewhalaw D, Asale A, Tushune K, Getachew Y, Duchateau L, Speybroeck $\mathrm{N}$. Bio-efficacy of selected long-lasting insecticidal nets against pyrethroid resistant Anopheles arabiensis from South-Western Ethiopia. Parasit Vectors. 2012:5:159.

23. Ethiopian Central Statistical Agency. Ethiopian population and housing census. Addis Ababa: Central Statistical Agency. 2007. http://catalog.ihsn. org/index.php/catalog/3583. Accessed 20 Nov 2015.

24. Mengesha T, Nigatu W, Ghiorgis W, Eshete $H$, Balcha F, Ishii A, et al. The 1991 malaria epidemic in Ethiopia, with reference to the outbreak in central Ethiopia. Ethiop J Health Dev. 1998;12:111-4.

25. Kenea O, Balkew M, Gebre-Michael T. Environmental factors associated with larval habitats of anopheline mosquitoes (Diptera:Culicidae) in irrigation and major drainage areas in the middle course of the Rift Valley, central Ethiopia. J Vector Borne Dis. 2011;48:85-92.

26. Kibret $\mathrm{S}$, Wilson GG, Tekie H, Petros B. Increased malaria transmission around irrigation schemes in Ethiopia and the potential of canal water management for malaria vector control. Malar J. 2014;13:360.

27. Bekele D, Belyhun Y, Petros B, Deressa W. Assessment of the effect of insecticide-treated nets and indoor residual spraying for malaria control in three rural kebeles of Adami Tulu District, South Central Ethiopia. Malar J. 2012;11:127. 
28. Gari T, Kenea O, Loha E, Deressa W, Hailu A, Balkew M, et al. Malaria incidence and entomological findings in an area targeted for a clusterrandomized controlled trial to prevent malaria in Ethiopia: results from a pilot study. Malar J. 2016;15:145.

29. Kloos H, Lindtjørn B. Famine and malnutrtion. In: Kloos H, Zein Z, editors The ecology of health and disease in Ethiopia. Boulder: Westview press; 1993.

30. International Federation of Red Cross Ethiopia. Drought-emergency plan of action operations update number 3 (MDRET0016). International Federation of Red Cross and Red Crescent Societies. 2015. http://reliefweb. int/report/ethiopia/mdret0016. Accessed 28 Jan 2016.

31. Agency Ethiopian National Meteorology. Adami Tullu and Zeway town annual meteorology data. Hawassa: Ethiopia Meteorology Agency; 2016.

32. Deressa W, Loha E, Balkew M, Hailu A, Gari T, Kenea O, et al. Combining long-lasting insecticidal nets and indoor residual spraying for malaria prevention in Ethiopia: study protocol for a cluster randomized controlled trial. Trials. 2016;17:20

33. Ethiopian Federal Ministry of Health. National malaria guideline. 3rd ed. Addis Ababa: Federal Ministry of Health. 2012. https://www.medbox.org/ national-malaria-guidelines-ethiopia/download.pdf. Accessed 15 Sept 2014

34. WHO. Guidelines for laboratory and field-testing of long-lasting insecticidal nets. World Health Organization. 2013. http://apps.who.int/iris/bitst ream/10665/80270/1/9789241505277_eng.pdf?ua=1. Accessed 23 Sept 2014.

35. NetWorks/Malaria Consortium. Long Lasting Insecticidal Net hole assessment facilitator's guide. Malaria Consortium. 2012. https://www.k4hea Ith.org/sites/default/files/lin_hole_assessment_facilitators_guide_revis ed_version_for_usaid_and_ccm_final.docx. Accessed 20 Sep 2014.

36. WHO. Vector control technical expert group report to MPAC on estimating functional survival of long-lasting insecticidal nets from field data.
Geneva: World Health Organization. 2013. http://www.who.int/malar ia/mpac/mpac_sep13_vcteg_llin_survival_report.pdf. Accessed 25 Oct 2014.

37. Vyas S, Kumaranayake L. Constructing socio-economic status indices: how to use principal components analysis. Health Policy Plan. 2006;21:459-68.

38. Howe LD, Hargreaves JR, Huttly SR. Issues in the construction of wealth indices for the measurement of socio-economic position in low-income countries. Emerg Themes Epidemiol. 2008;5:3.

39. Van Roey K, Sovannaroth S, Sochantha T, Touch MS, Pigeon O, Sluydts V, et al. A phase III trial to evaluate the efficacy, fabric integrity and community acceptance of Netprotect ${ }^{\circledR}$ using a recommended long-lasting insecticidal net as positive control. Malar J. 2014;13:256.

40. Mutuku FM, Khambira M, Bisanzio D, Mungai P, Mwanzo I, Muchiri EM, et al. Physical condition and maintenance of mosquito bed nets in Kwale County, coastal Kenya. Malar J. 2013;12:46.

41. VectorWorks. NetCALC. http://www.vector-works.org/resources/netca Ic-planning-tool/. Accessed 12 Feb 2016.

42. Loha E, Deressa W, Gari T, Balkew M, Kenea O, Solomon T, et al. Combining long-lasting insecticidal nets and indoor residual spraying for malaria prevention in Ethiopia: a cluster randomized controlled trial. In: ASTMH annual meeting. baltimore convention centre, Baltimore, USA. 2017.

43. Van der Hoek W, Konradsen F, Amerasinghe PH, Perera D, Piyarante MK, Amerasinghe FP. Towards a risk map of malaria for Sri Lanka: the importance of house location relative to vector breeding sites. Int J Epidemiol. 2003;32:280-5.

44. Staedke SG, Nottingham EW, Cox J, Kamya MR, Rosenthal PJ, Dorsey G. Short report: proximity to mosquito breeding sites as a risk factor for clinical malaria episodes in an urban cohort of Ugandan children. Am J Trop Med Hyg. 2003;69:244-6.
Ready to submit your research? Choose BMC and benefit from:

- fast, convenient online submission

- thorough peer review by experienced researchers in your field

- rapid publication on acceptance

- support for research data, including large and complex data types

- gold Open Access which fosters wider collaboration and increased citations

- maximum visibility for your research: over 100M website views per year

At BMC, research is always in progress.

Learn more biomedcentral.com/submissions 